\title{
Non-Invasive Ventilation in the Treatment of Acute Respiratory Failure with COVID-19
}

\author{
Nasibova EM* and Pashaev CN \\ Azerbaijan Medical University, Baku \\ *Corresponding author: Nasibova EM, Azerbaijan Medical University, Baku
}

\begin{abstract}
Decades have passed since the first reports of the successful use of mask non-invasive ventilation (NIV) in the treatment of respiratory failure. The aim of the study is determining the benefits of NIV in acute respiratory failure in patients with COVID-19. The study included patients admitted to the intensive care unit of the surgical clinic of the AMU from April 1 to May 1, 2020. NIV has visible advantages over mechanical ventilation. But it must be remembered that even in experienced hands, NIV is successful only in $75-90 \%$ of all cases, which depends on many factors, such as the severity of ONE, the training and experience of medical personnel. As with many types of therapy, operations, and technologies, improvement in the results of this method can be expected as experience is gained. High minute lung ventilation ( $>10 \mathrm{~L} / \mathrm{min}$ ) during NIV may predict non-invasive lung ventilation.
\end{abstract}

Keywords: Non-invasive ventilation; Acute respiratory failure; COVID-19

\section{Introduction}

Decades have passed since the first reports of the successful use of mask non-invasive ventilation (NIV) in the treatment of respiratory failure [1,2]. Attempts to use NIV with positive pressure in acute respiratory failure have been made earlier - in the 1970s and 1980s, but in general this experience was not very successful, because at that time devices for intermittent breathing with positive pressure were usually used (intermittent positivepressure breathing), which were poorly tolerated by patients and were usually intended for aerosol therapy [3]. The appearance of convenient masks for conducting ventilation with continuous positive airway pressure (CPAP) and new modes of respiratory support (especially pressure support mode) gave an impetus to the widespread introduction of NIV in clinical practice [4-6]. In 19902000 , the accumulation of experience with NIV and the encouraging positive results of this method in several studies allowed NIV to be assigned the first-line treatment place in acute respiratory failure [7-9]. The most important advantage of NIV in acute respiratory failure is the reduction in mortality, which may be associated with a reduced risk of nosocomial pneumonia and other hospital infections [10]. In addition, compared with invasive respiratory support, NIV is associated with less risk of damage and subsequent remodeling of lung tissue. Before using NIV, you need to pay attention to some important aspects of the method. The effectiveness of NIV depends on the correct assessment of its capabilities and limitations, while in order to avoid delays in the use of tracheal intubation and mechanical ventilation, in turn, the selection of a suitable patient, the participation of trained medical personnel and the timely detection of NIV failure are required [5,11,12]. Proper patient selection is a key factor in achieving NIV success. When COVID-19 is complicated by acute respiratory failure, patients with hypercapnia and moderate respiratory acidosis are most suitable, although a combination of respiratory and metabolic acidosis also lends itself well to NIV therapy [13]. Severe respiratory acidosis significantly increases the chances of patient intubation, especially at $\mathrm{pH}<7.20$ [14-17], but in routine practice, as experience shows, in some patients NIV can also be successfully performed at low $\mathrm{pH}$ values of 7.10 [18]. A coma condition is also a contraindication to NIV. As a rule, none of these factors is an absolute contraindication to NIV, but they should be taken into account when deciding on the beginning of NIV, as well as when ascertaining the inefficiency of 
the method and the need for tracheal intubation. Known predictors of NIV success or failure include the patient's neurological status (Glasgow scale), overall disease severity (APACHE II scale), and high tachypnea [19]. The "ideal" patient during the NIV should be a sufficiently communicative patient to provide conditions for applying and fitting the mask and synchronization with a respirator. Agitated and restless patients usually do not tolerate the NIV procedure. Most often in clinical practice, nasal or facial masks are used. The severity of the patient's condition may be a factor determining the appropriate type of mask: for example, patients with less severe respiratory failure (DN) are better adapted to nasal masks, which are more leaking when used, while in more severe situations, oronasal masks are better suited [20]. On the other hand, with the modern choice of various models of masks, individual characteristics and preferences of patients are considered. Tight fitting of the mask to the patient's face allows to minimize leakage and improve the patient's synchronization with the respirator. At the same time, with excessively tight contact of the mask with the patient's skin, ulcerations and necrosis may develop. In patients with agitation, anxiety, and high tachypnea, sedation may be prescribed to improve synchronization, but the risk of excessive sedation and respiratory depression should be remembered [19].

\section{The Aim of the Study}

Determining the benefits of NIV in acute respiratory failure in patients with COVID-19.

\section{Material and Research Methods}

The study included patients admitted to the intensive care unit of the surgical clinic of the AMU from April 1 to May 1, 2020.

\section{The Results of the Study}

Our experience with NIV has shown that most patients treated with NIV tolerate this procedure relatively well already at the initial stage. However, in several patients, during the first minutes or hours of NIV, no improvement (clinical indicators and gas exchange) is observed or the procedure is poorly tolerated, the proportion of such patients is usually about 15-35\%. Experience shows that longer attempts to use NIV without achieving a noticeable improvement only delay the time of tracheal intubation and mechanical ventilation, which significantly increases the risk of worsening respiratory failure, an unfavorable outcome, including death. Using NIV, we came to the conclusion that, in most cases, NIV therapy failures are detected quite early - on the first day from the initiation of respiratory support, however, in some patients, NVL therapy failure manifests itself later - 24-48-72 hours after the initial improvement. Lack of improvement in consciousness or respiratory acidosis 24 hours after onset is NIV another predictor of NIV failure. Indications for the implementation of NIV are as follows: a. Symptoms and signs of acute respiratory failure: a) severe shortness of breath at rest; b) $\mathrm{BH}>25$ / min, participation in the breathing of the auxiliary respiratory muscles, paradoxical breathing.

b. Signs of gas exchange disturbance: a) $\mathrm{PaCO}_{2}>45 \mathrm{~mm} \mathrm{Hg}$. Art., $\mathrm{pH}<7.35$; b) $\mathrm{PaO}_{2} / \mathrm{FiO}_{2}<200 \mathrm{mmHg}$. Art.

c. The exclusion criteria for NIV in ODN are as follows:

d. Stop breathing.

e. Unstable hemodynamics (hypotension, uncontrolled arrhythmias, or myocardial ischemia).

f. Inability to protect the respiratory tract (cough and swallowing disorders)

g. Excessive bronchial secretion.

h. Signs of impaired consciousness (agitation or oppression), the patient's inability to cooperate with medical personnel.

i. Facial trauma, burns, anatomical disorders that prevent masking.

Indications for the termination of NIV and the transition to intubation of the trachea and mechanical ventilation include the following:

i. The patient's inability to carry the mask due to discomfort or pain.

ii. The inability of the NIV to improve gas exchange within 2 hours: an increase or preservation of hypoxemia, despite the high values of PEEP and $\mathrm{FiO}_{2}$.

iii. Inability to mask ventilation to ease dyspnea.

iv. The need for endotracheal intubation to remove secretions or protect the respiratory tract.

v. Instability of hemodynamics and ECG, instability with the phenomena of ischemia or clinically significant ventricular arrhythmias.

vi. The increase in encephalopathy.

vii. During the study, the following advantages of non-invasive ventilation were identified:

viii. Prevention of "mechanical" and infectious complications associated with intubation, reducing the risk of developing infectious complications and mechanical damage (trauma to the larynx and trachea, stenosis, and bleeding from the upper respiratory tract).

ix. Preservation of natural protective reflexes of the upper respiratory tract.

x. Preservation of physiological cough, the patient's ability to talk, swallow, eat, cough up sputum. 
xi. Increase patient comfort.

xii. $\quad$ Reduced need for muscle relaxants, opioids, and sedatives.

xiii. The possibility of discrete use and weaning from the apparatus.

In our clinic, NIV was performed using Salvia Elisa ventilator respirators in $\mathrm{CPAP}+\mathrm{PSV}$ mode through a face mask. Used standard masks from Drager (Germany) or Respironics (USA). To determine the parameters of the gas and acid-base composition of the blood, an ABL500 gas analyzer with an OSM3 oximeter (Radiometer, Denmark) was used. Indicators of the function of external respiration were recorded from the display of the respirator. All data were recorded immediately before the start of ventilation. The level of PEEP and pressure support was set individually, based on the specific clinical situation. The ventilation parameters required by patients were as follows: PEEP - from 5 to $12 \mathrm{~cm}$ of water column, PSV - from 0 to $14 \mathrm{~cm}$ of water. column, $\mathrm{FiO}_{2}$ - from 0.3 to 0.6 . At the initial stage, auxiliary ventilation was carried out in a continuous mode. Further, a gradual decrease in respiratory support was carried out in accordance with the degree of clinical improvement, after which they switched to NIV sessions for several hours a day until it was completely canceled. The criterion for successful NIV was the improvement of the arterial blood gas composition and the ability to avoid endotracheal intubation.

\section{Clinical Case}

Patient - a man aged 34 years, with complaints of alternating chronic cough, temperature $39.2^{\circ} \mathrm{C}$, chills, headache, and shortness of breath. During auscultation of the lungs, crepitus was observed, moist rales in the lower lobes of the lungs, oxygen saturation $90 \%$, and respiratory rate 30-34 per minute. Admission laboratory tests included: WBC - 14,64 x 10\%/L, LYM - 0,00 x 109/L, albumin - 3.01 g/dL, PI - 50.5\%, PT - 14.8 sec., INR - 1.47, Fibrinogen - $128 \mathrm{mg} / \mathrm{L}$, GRP 47.4 mg/L, D-Dimer> 1500 ng/mL, Ferritin - 1178 ng/mL, P / F> 180. Chest $\mathrm{x}$-ray and CT are shown in the following (Figures 1-4).

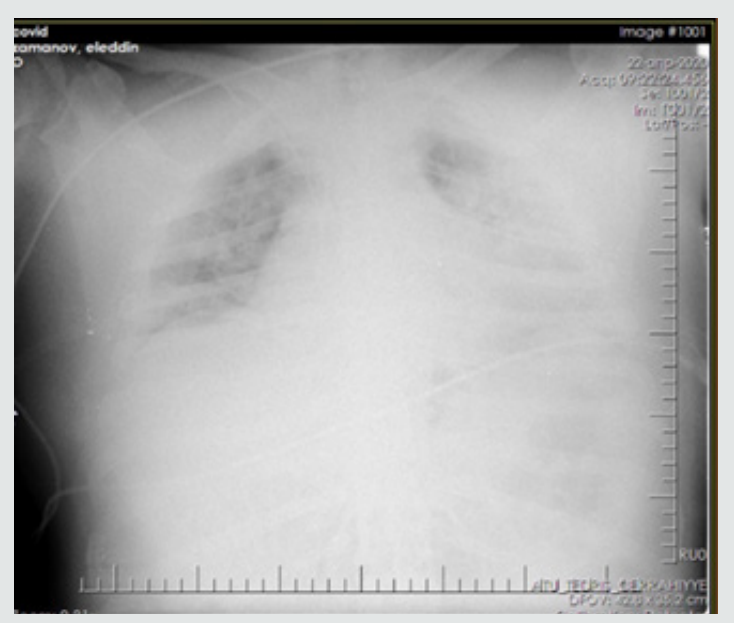

Figure 1: X-ray upon admission.

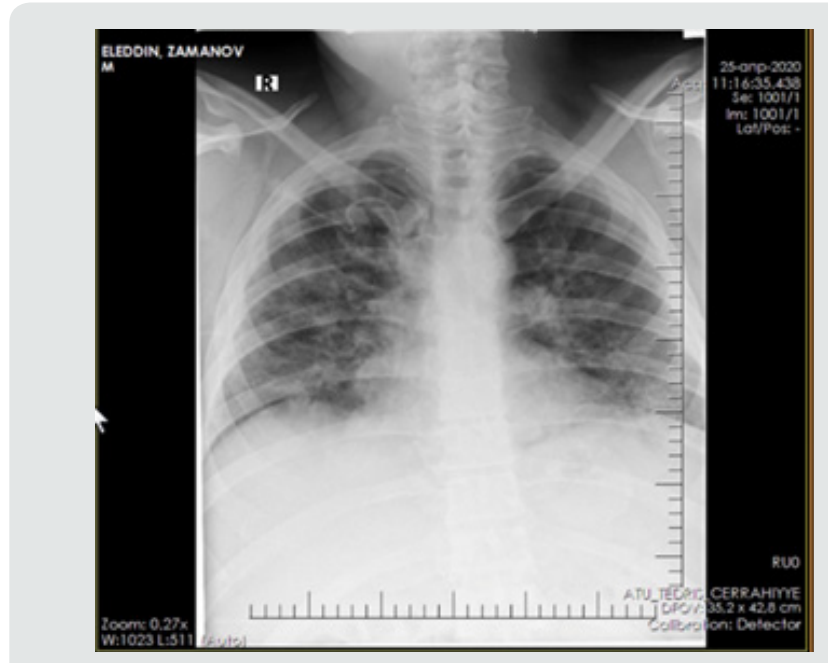

Figure 2: Radiograph after improvement of the patient.

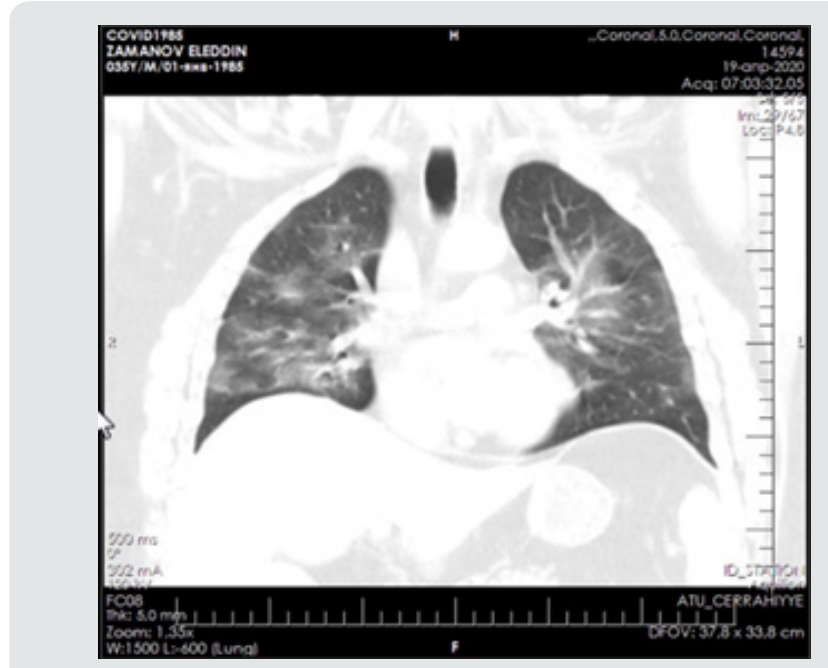

Figure 3: CT scan of the lungs upon admission.

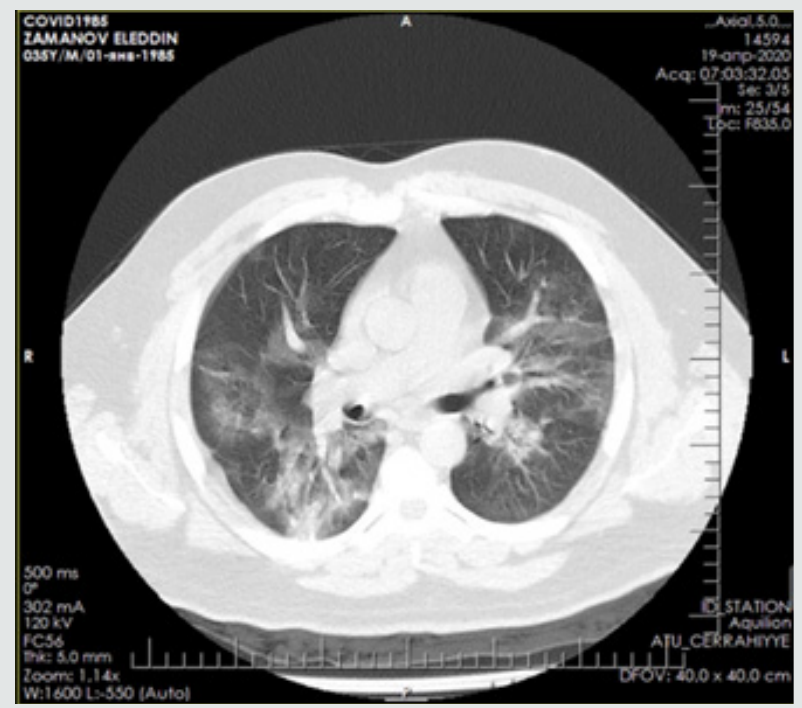

Figure 4: CT scan of the same patient. 
Non-invasive ventilation was carried out by an oral-nasal mask with a ventilator ELISA. Installation and adjustment of parameters was carried out according to the general condition and according to blood gas data: $\mathrm{BH}<35, \mathrm{pH}>7.30$, neurological dysfunction according to the Kelly scale> 3-5, a modified scale for determining the participation of auxiliary respiratory muscles $<3$ points. For hypercapnia, the following parameters were set Ps - 12, PEEP - $6 \mathrm{~cm}$ water column, $\mathrm{FiO}_{2}-30-40 \%$, and with hypoxemia - Ps - 12, PEEP - 5 $\mathrm{cm}$ water column, $\mathrm{FiO}_{2}-50-60$. The median treatment period with NIV was 6 days. The average daily treatment time with NIV on the first day was 16.5 hours, on the second day - 17.2 hours and on the third day 15.7 hours. The patient was discharged on the $14^{\text {th }}$ day with improvement.

\section{Conclusion}

1. NIV has visible advantages over mechanical ventilation

But it must be remembered that even in experienced hands, NIV is successful only in $75-90 \%$ of all cases, which depends on many factors, such as the severity of ONE, the training and experience of medical personnel. As with many types of therapy, operations, and technologies, improvement in the results of this method can be expected as experience is gained.

2. High minute lung ventilation ( $>10 \mathrm{~L} / \mathrm{min}$ ) during NIV may predict non-invasive lung ventilation.

\section{References}

1. Alraddadi BM, Qushmaq I, Al-Hameed FM, Mandourah Y, Almekhlafi GA, et al. (2019) Saudi Critical Care Trials Group. Noninvasive ventilation in critically ill patients with the middle east respiratory syndrome. Influenza \& Other Respiratory Viruses 13(4): 382-390.

2. Burns KEA, Meade MO, Premji A, Adhikari NKJ (2013) Noninvasive positive-pressure ventilation as a weaning strategy for intubated adults with respiratory failure. Cochrane Database of Systematic Reviews (12).

3. Dwarakanath A, Elliott MW (2013) Noninvasive ventilation in the management of acute hypercapnic respiratory failure. Breathe 9(5): 338-348.

4. Mehta S, Hill NS (2001) Noninvasive ventilation. Am J Resp Crit Care Med 163: 540-577.

5. Girou E, Brun Buisson C, Taillé S, Lemaire F, Brochard L (2003) Secular trends in nosocomial infections and mortality associated with noninvasive ventilation in patients with exacerbation of COPD and pulmonary edema. JAMA 290: 2985-2991.
6. Girou E, Schortgen F, Delclaux C, Christian BB, François B et al. (2000) Association of noninvasive ventilation with nosocomial infections and survival in critically ill patients. JAMA 284: 2361-2367.

7. Chandra D, Stamm JA, Taylor B, Rose MR, Lewis S, et al. (2012) Outcomes of noninvasive ventilation for acute exacerbations of chronic obstructive pulmonary disease in the United States, 1998-2008. Am J Respir Crit Care Med 185(2): 152-159.

8. Jois Bilowich P, Diercks D (2009) Emergency department stabilization of heart failure. Heart Fail Clin 5: 37-42.

9. Farha S, Ghamra Z, Hoisington ER, Butler RS, Stoller JK (2006) Use of noninvasive positive-pressure ventilation on the regular hospital ward: experience and correlates of success. Respir Care 51: 1237-1243.

10. Ram FS, Picot J, Lightowler J, Wedzicha JA (2004) Non-invasive positive pressure ventilation for treatment of respiratory failure due to exacerbations of chronic obstructive pulmonary disease. Cochrane Database Syst Rev (3): CD004104.

11. Schettino G, Altobelli N, Kacmarek RM (2008) Noninvasive positivepressure ventilation in acute respiratory failure outside clinical trials: Experience at the Massachusetts General Hospital. Crit Care Med 36: 441-447.

12. Zhou F, Yu T, Du R, Fan G, Liu Y, et al. (2020) Clinical course and risk factors for mortality of adult inpatients with COVID-19 in Wuhan, China: a retrospective study. Lancet, 2020. doi: 1016/S0140-6736(20)30566-3.

13. Lightowler JV, Wedzicha JA, Elliott MW, Ram FS (2003) Noninvasive positive pressure ventilation to treat respiratory failure resulting from exacerbations of chronic obstructive pulmonary disease: Cochrane systematic review and meta-analysis. BMJ 326: 185-189.

14. Nava S, Navalesi P, Conti G (2006) Time of non-invasive ventilation. Intensive Care Med 32: 361-370.

15. Nava S, Hill N (2009) Non-invasive ventilation in acute respiratory failure. Lancet 374: 250-259.

16. Peñuelas O, Frutos-Vivar F, Esteban A (2007) Noninvasive positivepressure ventilation in acute respiratory failure. CMAJ 177(10): 12111218.

17. Caples S, Gay PC (2005) Noninvasive positive pressure ventilation in the intensive care unit: a concise review. Crit Care Med 33: 2651-2658.

18. Peter JV, Moran JL, Phillips Hughes J, Warn D (2002) Noninvasive ventilation in acute respiratory failure: a meta-analysis update. Crit Care Med 30: 555-562.

19. Cuomo A, Delmastro M, Ceriana P, Stefano N, Giorgio C, et al. (2004) Noninvasive mechanical ventilation as a palliative treatment of acute respiratory failure in patients with end-stage solid cancer. Palliat Med 18: 602-610.

20. Deis JN, Abramo TJ, Crawley L (2008) Noninvasive respiratory support. Pediatr Emerg Care 24: 331-338.
To Submit Your Article Click Here:

This work is licensed under Creative Commons Attribution 4.0 License

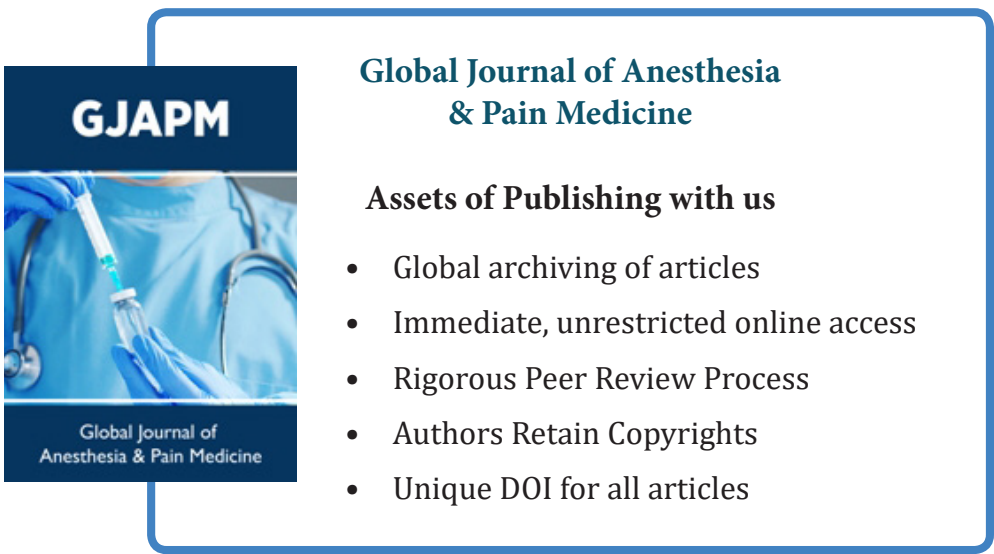

